\title{
Zytologie in der dermatologischen Praxis
}

Der Dermatologe Dr. Pierre Foss wurde im Rahmen der Fachtagung „Dermatologische Praxis“ in Frankenthal mit dem Innovationspreis Dermatologie ausgezeichnet. Mit der Auszeichnung fördert der Bundesverband der Deutschen Dermatologen (BVDD) gemeinsam mit der Professor Paul Gerson Unna Akademie Projekte, die den Behandlungserfolg von Patienten in der dermatologischen Praxis verbessern können.

Mit seinem Projekt „Zytoderm“ möchte Foss zytologische Methoden in der dermatologischen Praxis etablieren, um das diagnostische Potenzial verstärkt zu nutzen. „In der Wundbehandlung sind die Möglichkeiten, die wir dank zytologischer Technik haben, im- mens. So können beispielsweise gute und schlechte Wundbeläge klar voneinander abgegrenzt und entsprechend behandelt werden“, erklärt der Dermatologe.

Eine solide Aus- und Weiterbildung von Dermatologen im Bereich zytologischer Methoden bildet einen zentralen Aspekt von „Zytoderm“. Foss will die Zytologie fest in den Hautarztpraxen verankern und dafür Standardliteratur erarbeiten sowie Abrechnungsziffern etablieren. Der Saarländer engagiert sich bereits seit vielen Jahren in Bereich zytologischer Untersuchungen der Haut, insbesondere der Wundgrundzytologie. „Die Auszeichnung bestärkt mich und meine Kollegen wei- terhin im Einsatz für neuartige Entwicklungen, die unsere täglichen Behandlungserfolge in der Praxis verbessern können“, so Foss.

Der BVDD würdigt mit dem Preis gemeinsam mit der Professor Paul Gerson Unna Akademie zukunftsweisende Projekte in den Bereichen Praxismanagement, Vernetzung innerhalb der Fachgruppe und interdisziplinär, Diagnose und Behandlung sowie Praxisleistungen auch außerhalb der gesetzlichen Krankenversicherung. Die Auszeichnung ist mit einem Preisgeld von 5.000 Euro dotiert und nicht zweckgebunden. Die Auswahl des Preisträgers erfolgt auf Basis eines Kriterienkatalogs, der gemeinsam von allen Jury-Mitgliedern verabschiedet wird. Im Fokus stehen dabei direkte und indirekte Behandlungserfolge für Patienten.

Nach Informationen von Beiersdorf

\section{Wasserlöslicher Nagellack}

Für die topische Behandlung von Nagelmykosen sollte der Nagellack zwei Anforderungen erfüllen: fungizide und sporozide Wirkung aufweisen und den $\mathrm{Na}$ gel $\mathrm{zu}$ durchdringen vermögen. Die Kombination aus Ciclopirox und $\mathrm{HPCH}$ Lacktechnologie verfügt über diese Eigenschaften und überzeugt in Studien.

Nagelpilzerreger wie Trichophyton rubrum haben die unangenehme Eigenschaft, immer wieder zu kommen. Eine Quelle dieser Rezidive sind die im Nagelbett befindlichen, extrem widerstandsfähigen Pilzsporen. Wie Prof. HansJürgen Tietz vom Institut für Pilzkrankheiten und Mikrobiologie in Berlin betonte, stellen die Sporen ein zentrales Problem bei der Therapie der Nagelmykose dar und müssen unbedingt beseitigt werden, um Rezidive zu verhindern. Der medizinische Nagellack Ciclopoli ${ }^{\oplus}$ eignet sich sehr gut zur topischen Therapie von $\mathrm{Na}$ gelmykosen. Einerseits bekämpft der bewährte Wirkstoff Ciclopirox sowohl die Pilze als auch deren Sporen. Andererseits ermöglicht die patentierte Lacktechnologie $\left(\mathrm{ONY}-\mathrm{FLEX}^{\oplus}\right)$ mit dem Biopolymer Hydroxypropylchitosan (HPCH) einen raschen Transport des Wirkstoffs in den Nagel und das Nagelbett. Der wasserlös-

Im Nagelbett befindliche Pilzsporen können Quelle von Rezidiven sein.

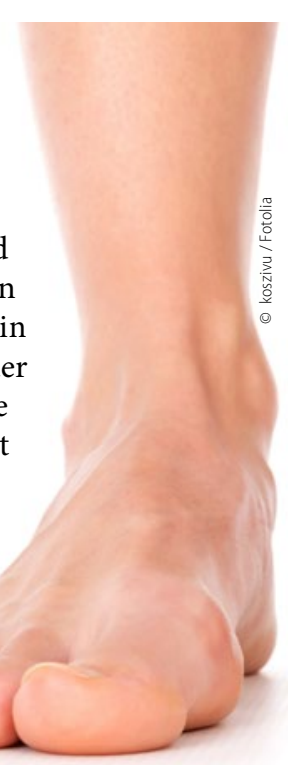

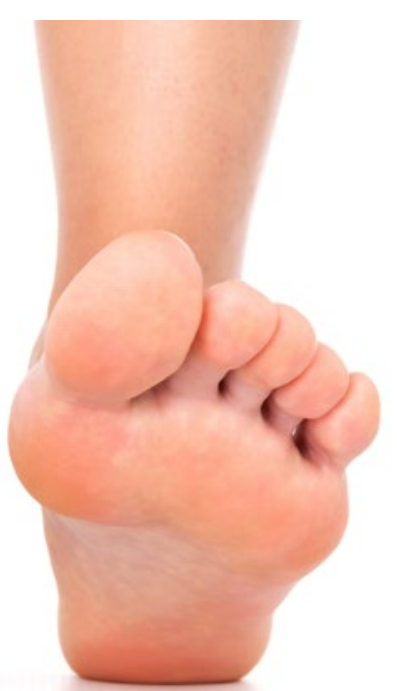

liche Lack wird abends aufgetragen und bildet einen unsichtbaren Film, der am nächsten Morgen einfach abgewaschen wird.

Eine randomisierte Studie verglich Ciclopoli Nagellack (täglich angewendet) mit einem handelsüblichen 5\% Amorolfin Nagellack auf Acrylatbasis (zweimal wöchentlich) bei 137 Patienten mit Onychomykose.

Nach zwölf Wochen erreichte Ciclopoli Nagellack das primäre Ziel der Studie die Nicht-Unterlegenheit bei der Umwandlung $\mathrm{zu}$ negativer Kultur. In der Ciclopoli-Gruppe wiesen 78,3\% der Patienten eine Konversion zu einer negativen Kultur auf gegenüber $64,7 \%$ in der Amorolfin-Gruppe. Nach 48 Wochen Behandlung zeigten mit $35,0 \%$ vs. $11,7 \%$ deutlich mehr Patienten der CiclopoliGruppe eine Komplettheilung (Konversion zu negativer KOH-Mikroskopie, negative Pilzkultur und $100 \%$ geheilter Nagel). Beim Therapieerfolg und der mykologischen Heilung war Ciclopoli ebenfalls signifikant überlegen (jeweils $\mathrm{p}<0,001)$ Dr. Marion Hofmann-Aßmus

Mykologie-Seminar „Dermatologische Kernkompetenz Nagelerkrankungen und Mykologie" im Rahmen der "Tagung Dermatologische Praxis", Frankenthal, 19. März 2017; Veranstalter: Taurus Pharma 